\title{
Mythos
}

Rivista di Storia delle Religioni

$13 \mid 2019$

Varia

\section{Le Germanie Segrete e il loro mito}

The Secret Germanies and their myth

\section{Carlotta Santini}

\section{(2) OpenEdition}

\section{Journals}

Edizione digitale

URL: http://journals.openedition.org/mythos/728

ISSN: 2037-7746

\section{Editore}

Salvatore Sciascia Editore

Notizia bibliografica digitale

Carlotta Santini, « Le Germanie Segrete e il loro mito », Mythos [Online], 13 | 2019, online dal 13 octobre 2019, consultato il 26 décembre 2019. URL : http://journals.openedition.org/mythos/728

Questo documento è stato generato automaticamente il 26 dicembre 2019.

Mythos 


\title{
Le Germanie Segrete e il loro mito
}

\author{
The Secret Germanies and their myth
}

\author{
Carlotta Santini
}

\section{La Germania Segreta: un topos letterario divenuto mitologema}

1 In una lettera famosa del 2 Febbraio 1965 indirizzata al mitologo ungherese Károly Kerényi, Furio Jesi parla di un progetto che aveva a cuore, e per il quale chiede consiglio e collaborazione al più anziano collega:

«Si tratta di un libro - che voglio intitolare Germania Segreta - destinato a studiare le sopravvivenze di talune immagini mitiche nella cultura tedesca del XIX e XX secolo. [...] Ella ha vissuto i problemi ivi proposti con la coscienza d'uno studioso del mito e insieme di uno spettatore non passivo della moderna civiltà europea. In particolare la sua partecipazione alla vicenda spirituale di Thomas Mann mi spinge a consultarla quale testimone più d'ogni altro sensibile alle gravi implicazioni morali dell'opera del maggiore artista tedesco del XX secolo». ${ }^{1}$

Nonostante il caveat di Kerényi che tentò di distogliere il giovane Jesi «da un progetto ciclopico non assolvibile con giustizia», ${ }^{2}$ Germania Segreta. Miti nella cultura tedesca del '900 è apparso nel 1967 per i tipi dell'editore Silva di Milano. Il compito ciclopico è stato dunque assolto, forse non tanto con giustizia, quanto con intelligenza, senza inciampare nei prevedibili ostacoli di una troppo scontata contro-retorica di sinistra, di un facile buonismo, o nelle duplici insidie del politicamente corretto.

Germania Segreta potrebbe essere definito senza tema di abusare di una metafora, un 'Viaggio in Germania', il Grand Tour di un intellettuale italiano, uso alle aperture cosmopolite, attraverso le regioni della cultura e della letteratura tedesche di OttoNovecento. In quest'opera, che per molti versi può essere definita un modello formale e di metodo per tutta la Germanistica italiana, Jesi ripercorre alcune delle tappe fondamentali del suo percorso di studioso della cultura tedesca. Nella costellazione di autori da lui scelta, da Goethe a Mann, a Bertolt Brecht, da Rilke a Wagner a Leo Frobenius, solo per citare i più significativi, Jesi illustra infatti alcune delle tappe 
fondamentali del processo di creazione e autocomprensione della cultura tedesca. Nella sua analisi, questa cultura appare percorsa, fin dai suoi inizi e nella sua interezza, da un'interna tensione, che si polarizza attorno a due tendenze opposte e apparentemente inconciliabili. Da una parte c'è l'ideale umanistico, classico, ecumenico, cosmopolita ed illuministico, come già lo conosceva il Settecento francese, ma più di questo percorso da una tensione verso la totalità, che non è semplice enciclopedismo, ma aspirazione alla comprensione generale ed ideale, intuitiva del mondo. Dall'altra abbiamo invece la tendenza al ripiegamento verso l'interno, l'interiorità, la ricerca dell'autenticità, del contenuto, il rifiuto della forma esteriore e il rifugiarsi nell'intimo ombroso ed arcano tipico della spiritualità tedesca di stampo protestante. Ma la Germania Segreta di Jesi non è che l'ultimo capitolo della lunga storia dell'idea della Germania segreta, che da almeno un secolo percorre la storia della cultura tedesca.

Quest'anima ancipite tedesca, ad esempio, è quella Doppelgesichtlichkeit di cui parla Norbert von Hellingrath (1888-1916), ${ }^{3}$ letterato e critico orbitante attorno al circolo di Stefan George, e a cui dava i nomi, ancora una volta, di due autori tedeschi : Goethe ed Hölderlin:

«Noi ci chiamiamo il 'popolo di Goethe', perché lo vediamo come ciò che di più alto possa raggiungere la nostra stirpe, come ciò che di più elevato sia cresciuto sul nostro ceppo, con la sua compiuta umanità, che costringe ad onorarlo perfino gli stranieri, i quali non possono intendere quel che vi è di più profondo in lui. Io dico che siamo il 'popolo di Hölderlin', perché fa parte del profondo dell'essenza tedesca, che il suo più intimo e ardente nocciolo, infinitamente al di sotto della crosta di scorie della superficie, venga alla luce solo in una Germania segreta ; e che si esterni in uomini che devono essere morti da lungo tempo prima di esse visti e di trovare eco, e in opere che affidano il loro segreto sempre solo a pochissimi, e ai più tacciono totalmente, e certo sono sempre inaccessibili a chi non sia tedesco; ché infatti tale Germania segreta è così certa del proprio intimo valore oppure così innocentemente inconsapevole del proprio significato, da non fare nessuno sforzo per essere udita, per esser veduta». ${ }^{4}$

Da una parte dunque una Germania che si manifesta nella dimensione luminosa del visibile, dell'estensione, della vastità degli orizzonti di Goethe, che, come dirà Friedrich Nietzsche nelle Scorribande di un Inattuale, persegue la totalità attraverso la misura di un orizzonte di senso. ${ }^{5}$ A questa dimensione estensiva, solo apparentemente tracotante dello spirito tedesco, si contrappone una dimensione intensiva, che scava nel profondo, una dimensione verticale e stavolta sì vertiginosa. Questa dimensione intensiva dello spirito e della cultura tedesca può esprimersi a sua volta secondo tre vettori : quello dell'interiorità e della spiritualità introspettiva di stampo luterano; quello della ricerca di un ideale trascendentale, laddove la profondità diviene elevazione e si incarna nell'esperienza mistica di un Meister Eckhart e di Ildegarda di Bingen; per finire il vettore storico, che proietta una verticale nel passato per favorire un ritorno all'origine, all'Ursprung. Tutte queste dimensioni, come anche le polarità illustrate da von Hellingrath, saranno egualmente presenti nel mitologema della Germania Segreta.

Passando ad un meta-livello di analisi, che intendo mantenere nel corso di questo mio contributo, io definisco la Germania Segreta innanzitutto come un topos letterario, nel senso più proprio dato a questo termine da Ernst Robert Curtius nel suo libro sulla Letteratura europea e Medioevo latino. ${ }^{6}$ Si tratta infatti di un fenomeno in origine eminentemente letterario, che si sviluppa all'interno di una cultura letteraria, quella tedesca. All'interno di questa stessa cultura, il topos della Germania Segreta viene in un primo momento mobilitato come strumento critico per mutarsi poi, o piuttosto 
riconoscersi, in un vero e proprio mitologema, che viene prima interiorizzato ed in seguito giudicato. Questa formula binaria, la Germania Segreta, che allude ad una realtà in prima istanza letteraria, si carica presto di significati che esulano dalla dimensione puramente finzionale e finanche estetica, e ambisce ad una validità più amplia, sia in campo storico, che sociale e politico, della cultura e della Weltanschauung tedesca.

7 Convenzionalmente si ritiene che l'espressione Geheimes Deutschland sia stata coniata da Stefan George, che intitola così nel 1922 una delle sue poesie più ermetiche. Di fatto le prime attestazioni scritte di questa formula sono da rintracciarsi in altri testi di autori orbitanti attorno al circolo di George, come il sopra menzionato Norbert von Hellingrath che la usa già nel 1915 negli scritti su Hölderlin. Karl Wolfskehl (1869-1948), poeta e letterato di origini ebraiche, nel 1910 utilizzò quest'espressione nel volume inaugurale dello Jahrbuch für die geistige Bewegung, ${ }^{7}$ per definire l'intera poetica di George. Ma le radici di questo concetto possono essere rintracciate anche nelle opere di Julius Langbehn (1851-1907) e di Paul de Lagarde (alias Paul Bötticher 1827-1891), quest'ultimo che nel 1878 così definiva la Germania del suo ideale:

«L'antica Germania non ha più eredi : ma si trova molto più in profondità e molto più in alto di dove la cercano l'attuale Cancelliere del Reich e i suoi amici. Ciò che viene gettato come metallo risonante nel crogiolo per la fusione delle campane del futuro non ha nulla a che vedere con la stampa e ancora meno con la stampa governativa. È dietro l'aratro e nella foresta, che si troveràm. ${ }^{8}$

Come fu concepita da George ed illustrata nelle stanze del suo componimento, la Germania Segreta è un luogo dello spirito, i cui confini si estendono al di là del tempo e dello spazio, ma che sostanzialmente coincidono, nella sua intenzione, come già nell'analisi di Wolfskehl, con i confini della sua poetica. Essa si oppone, come custode dell'immaginario poetico dell'autore e del suo ideale umanistico, alla Germania pubblica, alla Germania ufficiale, alla prosaicità dei borghesi tedeschi, dei campi di patate e di rape, della politica del cancelliere del Reich.

9 Se volessimo però definire in che cosa consista la Germania Segreta, il nostro compito sarebbe ben arduo. Nel componimento di George, così come in tutte le altre attestazioni che prenderemo in esame in questo contributo, la Germania Segreta non riceve mai un contenuto positivo. Essa si definisce per ciò che non è : secondo cioè una retorica $a$ contrario che sfrutta la particolare sacralità della teologia negativa. Quando dei contenuti sembrano mobilitati nell'orbita del concetto di Germania Segreta, questi contenuti si disporranno nei non luoghi del ricordo, dell'ideale, del passato, della proiezione del futuro : ad ogni modo, nella dimensione dell'inattualità e dell'irrealtà. È in questi non luoghi, in questo spazio senza tempo, che si produce laddove si esperisce una mancanza (l'assenza di Dio, la mancanza di senso, la carenza di volontà) che si sviluppano i miti, secondo la celebre tesi di Furio Jesi. ${ }^{9}$

10 In questo senso, la Germania Segreta, creata dagli intellettuali e per gli intellettuali, si rivela da topos letterario, un topos mitico, laddove il panorama intellettuale tedesco fin de siècle vive la sua più profonda crisi di senso, nello scoprirsi radicalmente disconnesso dal tessuto storico e politico della Germania presente. La stessa formula, la Germania Segreta, diviene invocazione, titolo, slogan. Come parola d'ordine che si passano di mano in mano gli adepti del circolo di George, e che ognuno modifica a suo talento, il concetto negativo di Germania Segreta assume i connotati di una di quelle 'idee senza parole' contro le quali Jesi metteva in guardia nel suo Cultura di Destra. ${ }^{10}$ Siamo qui di fronte ad un'idea troppo piena, ma al contempo vuota, sufficientemente vaga per accogliere i più diversi significati, e come vedremo, i più diversi giudizi e le più diverse 
sfumature. In questo articolo cercherò di definire i confini concettuali della Germania Segreta, seguendo alcune delle fila della formazione e dello sviluppo di questa idea. In questa analisi mi avvarrò, tra le altre, della guida metodologica di Jesi, che costituisce non solo un contributo critico sulla storia di questo concetto, quanto esso stesso un capitolo a sé di questo processo culturale. Verranno discussi infatti alcuni degli autori che hanno contribuito alla prima formulazione come topos letterario del concetto di Germania Segreta e al suo sviluppo come mitologema: tra gli altri Paul de Lagarde ed Ernst Kantorowicz. Ma troveranno posto in questa analisi anche quegli autori, come lo stesso Jesi, e come Gaston Choisy, che opponendosi più o meno criticamente al concetto di Germania Segreta, costituiscono a loro volta un momento ineludibile nella storia di questa idea.

\section{Germania Segreta o Germanie Segrete?}

11 Il presupposto della Germania Segreta è che esistono molte Germanie. Come minimo, infatti, ne esistono due, una segreta ed una non segreta. Ma le Germanie che collaborano a costruire il concetto negativo di Germania segreta sono molte, come sostiene Gaston Choisy in incipit al suo pamphlet del 1915, l'Allemagne Secrète:

«C'è la Germania dei politici e dei militari, c'è la Germania delle università e dei laboratori, c'è la Germania degli ingegneri, dell'industria, del commercio e della finanza, persino degli scrittori e degli artisti: queste Germanie si possono forse imparare dai libri. "Ci sono pensieri e gesti in Germania che vanno oltre ogni quadro e che nessuna formula può rinchiudere, che sono di tutte le categorie e di tutti i mestieri e professioni e che non possono essere visti o espressi da nessuna parte o che possono essere visti solo per caso ed espressi solo dall'oblio, che finalmente per loro stessa natura si allontanano nel segreto che le oscure manovre dei nostri nemici hanno preparato e condotto alla Grande Guerra"». ${ }^{11}$

Come si può notare, il passaggio appena trascritto contiene una doppia citazione. Questo produce un'ambiguità voluta da parte di Choisy, a cui appartengono infatti solo le prime righe, dove si elencano le varie Germanie che, secondo la scettica affermazione dell'autore, si possono apprendere dai libri. Gaston Choisy è stato un pubblicista francese, autore di numerosi reportages sulla Grande Guerra, che può essere annoverato a buon diritto tra quei "nemici" del popolo tedesco citati nella seconda parte del suddetto passaggio. "Ce livre n'est pas sans haine», ${ }^{12}$ come dichiara lo stesso Choisy poche righe più avanti, e certo questo è il meno che si possa dire di un libro come l' Allemagne Secrète, aspro e violento, a tratti miserabile (soprattutto nei misogini passaggi sulla Femme allemande o il capitolo Au pays di stupre, du sadisme et de la métaphysique) che risente del clima politico anti-boches della Francia del primo conflitto mondiale.

Questo libro, per esplicita dichiarazione dell'autore, ha come scopo quello di "smantellare 25 anni di menzogna sulla Grandeur tedesca», ${ }^{13}$ e lo fa con un misto di intelligenza e volgarità, di lucidità e faziosità, restituendo in forma caricaturale $i$ modelli della cultura e della letteratura tedesca. Il libro di Choisy, con la sua critica della Germania Segreta, costituisce quasi una falsariga dell'omonimo volume di Jesi del 1967. Sebbene non mi risultino citazioni dirette di Choisy e manchi la prova di una effettiva lettura di questo testo, da parte di Jesi, alcune somiglianze formali e strutturali nelle opere di questi due autori potrebbero rendere una tale ipotesi quantomeno percorribile. Alcuni argomenti affrontati da Jesi nella sua Germania Segreta (l'immagine della donna tedesca, il prestigio dell'intellettuale, la condiscendenza verso il pensiero 
esoterico) seguono un ordine molto simile a quello dei capitoli di Choisy (La femme allemande, Le dogme du silence, Leur Archisecret). Nella Germania Segreta di Choisy si attacca la Weltanschauung tedesca, che viene messa in scena (ed in ridicolo) attraverso i suoi letterati e artisti e le figure di finzione da loro concepite (come la Gretchen del Faust o la Lotte del Werther). Come anche nel caso di Jesi, il profilo di questi prodotti della cultura tedesca è delineato da Choisy con acume e senso critico, condito però in questo caso da un'esplicita verve dissacratoria con finalità caricaturali.

Le "menzogne sulla Grandeur tedesca" sono quelle esposte nella seconda parte del già citato passaggio dell'incipit di Choisy, che, come avevo anticipato, è a sua volta una citazione letterale. Si tratta di una citazione da un libro di Adolf Lasson (1832-1917), filosofo tedesco di origini ebraiche, Das Kulturideal und der Krieg (LASSON 1868). Già in questo testo così precoce, scritto poco prima della Guerra Franco-Prussiana (1870-71) da un sostenitore della causa nazionale tedesca, troviamo l'argomento della distinzione delle molte Germanie. Lasson invita i "nemici" del popolo tedesco (i Francesi e il resto d'Europa) a tenere distinta la Germania dello spirito da quella militante e militare, ed anzi a riconsiderare il giudizio storico e politico sulla Germania in virtù del valore di questa sua anima inespressa ed inesprimibile. Lasson è considerato da Choisy come il precursore di quei celebri 93 firmatari dell'Aufruf an die Kulturwelt (1914), l'appello degli intellettuali tedeschi ${ }^{14}$ ai loro contemporanei europei alla vigilia della I Guerra Mondiale, che mirava, come anche Lasson cinquant'anni prima, a risollevare la reputazione della Germania e, idealmente, a contribuire a scongiurare l'entrata in guerra dei paesi ancora neutrali.

La Germania di cui parla Lasson, contiene in nuce già tutti gli elementi che saranno quelli della Germania Segreta di George e di von Hellingrath. Essa è una Germania di gesti e di pensieri, invisibile ed inesprimibile. Vi troviamo già enunciato l'elemento dell'incommensurabilità, dell'elevazione estatica, dell'essere al di là di ogni definizione possibile (un'idea senza parole), come anche l'elemento misterico del segreto, dell'oscurità, dell'oblio dell'origine. Giocando sull'aspirazione di chi, come de Lagarde e Lasson, rivendica il primato di una Germania spirituale e culturale, le cui radici affondano nell'antichità e contraddicono la fin troppo imperfetta realtà storico-politica del neonato stato tedesco, Choisy afferma, tanto più decisamente, che da sempre esiste una ed una sola Germania :

«Perché la Germania essenziale, la Germania che permane sotto le forme che passano e la vergogna che si dimentica, era già interamente presente nel caos del tempo in cui la Germania non aveva nome, nel mistero delle foreste che la mano dell'uomo è stata così lenta a dissodare, e nella desolazione delle paludi che il sole non ha finito di prosciugare ; la Germania è nata dall'incontro di questo mistero e di questa desolazione e in essa sono identificabili quei tratti che l'imparentano con $\mathrm{i}$ rettili e le grandi fiere amiche dei bassifondi e del fitto sottoboscom. ${ }^{15}$

Ma al di là della ben motivata haine di Choisy, qui tocchiamo con mano quello che è il vero punto sensibile del dibattito sulla Germania Segreta, un dibattito che non a caso si è di volta in volta riattivato : prima della Guerra Franco-Prussiana, prima della Grande Guerra, tra le due guerre e nell'immediato secondo dopoguerra. Lo snodo cruciale della questione si gioca sulla possibilità o meno di distinguere tra molte Germanie, sulla legittimità di concepire una cesura, e dunque di separare il giudizio, tra una Germania della cultura, dei letterati, degli intellettuali, dello spirito, e una Germania imperialista, egemonica, economica, moderna, tecnicista. Se Choisy muoveva agli intellettuali tedeschi l'accusa di falsità e di cripto-imperialismo nello sbandierare la cultura tedesca 
come un feticcio per garantire la menzogna di un volto civile ad una Germania altresì guerrafondaia, Jesi sposterà la questione su un piano certo meno polemico, ma non per questo meno decisivo, quello morale. Anche Jesi è convinto che il sogno di una Germania Segreta non sia che un alibi per gli intellettuali tedeschi, per quanto forse, in alcuni casi, un alibi creato in buona fede. Quel che interessa Jesi è di scoprire perché gli intellettuali tedeschi hanno dovuto crearsi questo alibi, perché dalla fine dell'Ottocento e per tutto uno dei secoli più controverso della storia tedesca, essi abbiano avuto bisogno del mito della Germania Segreta.

L'argomento della "distinzione" delle Germanie sarà centrale anche nel caso di uno degli attori più celebri del dibattito sulla Germania Segreta: lo storico Ernst Kantorowicz (1895-1963). Come già Lasson e Wolfskehl, anche Kantorowicz appartiene a quella intelligentia di intellettuali tedeschi di origini ebraiche che abbracciano posizioni conservatrici e nazionaliste. Con l'avvento delle leggi razziali in Germania nel 1933, benché il suo prestigio lo tenesse ancora al riparo da ritorsioni, Kantorowicz si ritirò per protesta dall'insegnamento per la durata del semestre estivo. La simbolica brevità di questa protesta e l'immediato ritorno all'insegnamento nel semestre successivo avevano per Kantorowicz lo scopo preciso di riappropriarsi di uno spazio pubblico ed istituzionale, quale quello dell'Università, che le leggi razziali minacciavano di negargli. Nel bellissimo discorso che tenne in occasione del suo rientro (Frankfurt am Main, 14 Novembre 1933, v. KANTOROWICZ 1997), ${ }^{16}$ intitolato non a caso La Germania Segreta (Das Geheime Deutschland), Kantorowicz scelse di ribadire la ben nota distinzione delle due Germanie, innalzando la Germania dello spirito e delle Università contro le ombre e le miserie della Germania nazista. Una conferenza sulla Germania Segreta tenuta in quanto Professore - prima ancora che in quanto reduce di guerra, ebreo tedesco e nazionalista - in una Università tedesca significava per Kantorowicz tenere saldamente e ostentatamente il piede nell'istituzione pubblica e secolare che questa stessa Germania Segreta era chiamata a rappresentare.

Al di là del fallimento di fatto di questa impresa, ${ }^{17}$ è la legittimità stessa della distinzione - che come vediamo continua ad operare in Kantorowicz - unitamente alla volontà di mantenere il giudizio intellettuale sulla cultura tedesca quanto più possibilmente distinto da quello etico nei confronti della Germania nazista, ciò su cui Jesi appunterà la sua critica. La sospensione del giudizio morale per la "Germania Segreta" rischia per Jesi di offuscare non tanto la ovvia condanna per la Germania nazista, quanto la presa di coscienza delle responsabilità culturali della intelligentia tedesca, degli artisti e degli intellettuali, indipendentemente dal loro commitment nazionalista. Non ci si può accontentare di un processo alla Germania pubblica, fin troppo facile nel caso delle violenze della II Guerra Mondiale, senza comprendere i motivi culturali che hanno fatto sì che la nazione più evoluta e culturalmente raffinata del XIX secolo sia stata la portatrice del messaggio antisemita e delle atrocità del terzo Reich. Anche per Jesi, come per Choisy, non si può parlare di molte Germanie, ma di una sola, benché in sé scissa e irrisolta.

\section{La Germania che non c'è}

Se le pretese Germanie sono dunque molte, la Germania Segreta ne presuppone sempre almeno un'altra, un Doppelgänger al quale è legata da un rapporto di opposizione polare: una Germania pubblica contro una Germania segreta, una reale contro una ideale, la 
Germania legata alla storia e quella invece eterna, una Germania visibile ed una invisibile, una materiale ed una spirituale, una esteriore ed una interiore. Tutte queste opposizioni si prestano secondo Jesi ad una fin troppo facile traduzione nella distinzione, al contempo fuorviante e semplicistica, tra una Germania buona ed una cattiva. Nel resto di questo articolo analizzerò alcune di queste coppie polari e ne metterò in luce le implicazioni culturali nell'articolazione del mitologema della Germania Segreta.

20 La perduta e ritrovata città di Wagadu, di cui parla un racconto africano riportato dall'antropologo Leo Frobenius (1873-1938) e ripreso da Jesi nel suo Letteratura e mito, costituisce un ottimo canovaccio per illustrare i caratteri salienti della Germania Segreta:

«Il liuto di Gassire: "Quattro volte Wagadu apparve meravigliosa nella luce del giorno, quattro volte andò perduta, così che gli uomini non la videro... ogni volta che Wagadu era eretta sulla terra, visibile agli uomini, aveva quattro porte, una verso il nord, una verso l'ovest, una verso l'est, una verso il sud. [...] non importa se Wagadu è fatta di pietra e terra, o se vive solo come un'ombra nel pensiero e nella nostalgia dei suoi figli. Poiché Wagadu per sé non è di pietra, non è di legno, non è di terra. Wagadu è la forza che vive nel cuore degli uomini, ora riconoscibile, perché gli occhi permettono di riconoscerla, perché le orecchie sentono i colpi di spada e i suoni sugli scudi, e ora invisibile, perché stanca e incalzata dall'indomabilità degli uomini si addormenta». ${ }^{18}$

21 La mitica città di Wagadu cantata nel Liuto di Gassire, che tanto incantò il poeta Ezra Pound, ${ }^{19}$ è invisibile agli occhi di tutti, se non di coloro che vogliono vederla. E' al contempo reale ed irreale, e non importa se sia fatta di pietra e terra o dell'etere dei pensieri: essa vive nel ricordo vivo dei suoi abitanti, realtà eterna e perenne. Essa è al di sopra dei popoli e degli individui che, anche se talvolta sono chiamati a rappresentarla, per via della loro indomabilità non sono degni di possederla durevolmente. Gli uomini chiamati ad abitare la Germania Segreta, come gli uomini di Wagadu, sono i portatori di una cultura, nel senso puramente antropologico del termine: essi sono i vettori (Träger è il termine utilizzato dagli antropologi della scuola di Berlino nell'Ottocento, bearer il termine oggi adottato dalla contemporanea cultural anthropology), i veicoli che trasportano la cultura, mentre questa li sopravanza e di fatto gode di uno statuto indipendentemente da loro.

22 La mitica Wagadu ha quattro porte orientate verso i quattro punti cardinali. Essa rappresenta dunque un cosmogramma, come molti se ne ritrovano nelle pagine di studi morfologici di Frobenius. Ma quali sono i confini, i punti di riferimento dell'orizzonte della mitica Germania Segreta? Secondo il discorso di Francoforte di Kantorowicz, non solo i confini politici sono irrilevanti, ma anche quelli geografici. Agli occhi dell'umanista (che elegantemente soprassiede sui prosaici campi di rape), ${ }^{20}$ la geografia della Germania Segreta contiene tutto il mondo: essa è un po' Toscana, un po' Francia, un po' Grecia e un po' Sicilia. Se le sue coordinate geografiche ideali abbracciano l'Europa intera, ancor più difficile sarà definire la nazionalità dei suoi abitanti. Come Imperatori della Germania Segreta sono designati tutti quegli spiriti che hanno contribuito a costruirne l'idea, indipendentemente dalla loro "germanicità" o meno, in tutti i tempi e in tutti i luoghi. Al posto di uomini politici e conquistatori abbiamo dunque santi, artisti, visionari e pensatori. Tutte le figure che compongono il Walhalla della Germania Segreta sono di fatto delle figure eccentriche, nel senso che tracciano delle vie centrifughe rispetto alla ristretta concezione di un popolo, di un suolo e di una 
nazione tedesca. Nel novero degli imperatori segreti di questa Germania, incontriamo dunque Albrecht Dürer, che per primo insegnò lo stile italiano all'Europa del Nord, Holbein il Giovane, che visse tra la Svizzera e Londra; abbiamo poi Lutero, che inventò una lingua che non c'era e aprì ai tedeschi le porte della spiritualità interiore; il mago Hamann che abitava ai confini dell'impero (nella Königsberg che oggi è divenuta Kaliningrad) e il suo allievo Herder, che passò lunghi anni a Riga nei domini dello Zar. Terminano la serie Winckelmann, che visse in Italia e morì a Trieste, Hölderlin poeta del mondo greco, fino al grandissimo Goethe, che voleva imparare l'inglese e non riconosceva altri confini se non quelli dell'orizzonte del suo sguardo interiore. Sembra quasi che il vero spirito tedesco sia irrimediabilmente estraneo alla Germania, e che quanto più da lontano la si guardi, tanto più essa acquisti coerenza e significato.

23 Come campione di questo stuolo di imperatori ideali, troviamo finalmente un imperatore reale: Federico II di Svevia, lo Stupor Mundi al quale Kantorowicz ha dedicato la sua opera capitale nel $1927 .{ }^{21}$ Più che un imperatore terreno del Sacro Romano Impero, lo Svevo Federico, nato a Jesi nelle Marche, vissuto tra la Sicilia e le Puglie, amico del Saladino, che solo un volta in vita sua mise piede in Germania, rappresenta per Kantorowicz un'idea di regalità e cultura. Federico II è per lo storico tedesco un grande artista che ha saputo coniare forme originali, che ha creato una cultura cosmopolita che ha connotato un'epoca ed ha lasciato un segno indelebile nella Weltanschauung medioevale. ${ }^{22}$

24 Non c'è dunque un popolo reale della Germania Segreta, ma solo uno ideale. Di esso fanno parte i grandi del passato, unitamente agli intellettuali del presente, anche questi, per altro, rappresentati di un'idea di germanesimo allargata (molti degli intellettuali che abbiamo citato in questo contesto vantavano origini ebraiche). $\grave{\mathrm{E}}$ questo il vero significato dell'Urvolk di fichtiana memoria, di cui parlano i teorici della Germania Segreta: non il leggendario popolo germanico che l'archeologo Gustaf Kossinna (1858-1931) ${ }^{23}$ credeva di poter riconoscere già stanziato in Germania fin dal Neolitico; non quei Germani di cui parla Tacito, e tantomeno i Prussiani moderni, o i Nazisti futuri. L'Urvolk è quel popolo che non è ancora nato, ma il cui modello è già presente da sempre nella contemplazione degli intellettuali tedeschi. Esso è un ideale dotato di un potenziale operativo. Se vogliamo seguire la tesi di Kerényi, col quale Jesi aveva discusso questi temi nel loro già citato epistolario, ${ }^{24}$ l'Urvolk è un mito, poiché costituisce un modello, un ideale regolativo al quale ci si ispira e sul quale ci si appoggia per muoversi nel presente ed agire sul futuro. In questo l'Urvolk della Germania Segreta non è dissimile dall'Übermesch di Friedrich Nietzsche, che a sua volta tradisce la sua origine di teologoumeno cristologico. ${ }^{25}$ L'unica differenza, se si vuole, è che la proiezione dell'Urvolk si fa in un passato originario, mentre l'Übermesch si situa in un futuro indefinito, benché di fatto la loro natura di modelli tipologici li ponga entrambi sul piano del presente fuori dalla storia.

25 Paul de Lagarde, non certo il meno politicamente coinvolto dei teorici dell'ideale germanico, è esplicito in questo senso:

«La Germania non è un concetto geografico, né politico nel senso comune del termine. Una patria [Vaterland] appartiene al novero delle forze etiche, e quindi i suoi affari non possono essere curati dalle classi dirigenti, ma solo dal pathos etico di tutti i suoi figli». ${ }^{26}$ perduta Wagadu, con lo spirito e le aspirazioni di tutti gli individui che ne costituiscono il corpo. La modalità di appartenenza dei singoli individui a questo corpo spirituale ed 
ideale trova una sua teorizzazione nella ripresa della dottrina cristiana del corpo mistico da parte di Kantorowicz nel suo celebre saggio I due corpi del re. ${ }^{27} \mathrm{La}$ Germania Segreta non è uno stato con dei confini e degli abitanti reali, non è nemmeno un'entità politica nel senso del corpo collettivo che organizza gerarchicamente le membra che lo compongono (come nella visione del Leviatano di Hobbes). Essa è un'idea eterna, che si mantiene al di sopra degli eventi, e che è capace, con le sue incarnazioni o con il suo esempio, di conferire senso a chi vi si appelli.

Ciononostante la Germania Segreta può essere una patria, un Vaterland secondo de Lagarde, ma forse potremmo utilizzare qui piuttosto il concetto heideggeriano di Heimat, più adatto a comprendere il tipo di appartenenza degli abitanti della Germania Segreta. Non si tratta infatti di un'appartenenza come quella che unisce gli uomini in una sola fede (anche politica) e che li spinge ad una coesione reale, ad eliminare le differenze e $\mathrm{a}$ - per così dire - fare quadrato dirigendosi tutti insieme, come un corpo unico, verso chissà quale meta dell'avvenire. Questa Heimat è, come vuole l'Heidegger dei saggi su Hölderlin ${ }^{28}$ un invito all'Heimkehr, al ritorno a sé, alle proprie origini intese come proprio nucleo di senso. Il movimento di aspirazione verso la Germania Segreta è dunque inizialmente un movimento individuale, che parte dalla coscienza del singolo: e di fatto forti, distinte e granitiche individualità sono quelle degli intellettuali che hanno contribuito alla configurazione di questo mitologema.

Un secondo movimento, quello generale, che vede coinvolta una comunità che si orienta verso un'unità, che può anche essere nazionale, è del tutto accessorio, ed anzi a ben vedere sarebbe escluso di principio dai teorici della Germania Segreta. Ancora de Lagarde:

«La Germania che amiamo e desideriamo vedere non è mai esistita, e forse non esisterà mai. L'ideale è qualcosa che è e non è allo stesso tempo. È il sole che risplende nel cuore più profondo degli uomini, attorno al quale ruotano i nostri pensieri e le nostre forze, attorno al quale ruotano tutti i centri, che orbita intorno alla nostra vita, un sole il cui bagliore si affievolisce sempre di più quando emerge dalle profondità delle anime alla luce del giorno». ${ }^{29}$

L'insistenza sulla mancanza di un corpo politico reale, sull'indeterminatezza geografica e temporale della Germania Segreta, non può non essere considerata a sua volta un'esplicita presa di posizione politica. La Germania, per quanto verspätete Nation par excellence, ${ }^{30}$ con il collante dell'egemonia prussiana riaffermatasi con la fondazione del II Reich a seguito della Guerra Franco-Prussiana, poteva essere considerata, alla fine dell'Ottocento, oramai una realtà. La posizione di de Lagarde può dunque essere definita anti-politica, ma non ancora a-politica come sarà quella di George o la presunta posizione im-politica di un Thomas Mann tra le due Guerre. ${ }^{31}$ De Lagarde fu infatti molto attivo politicamente all'interno del partito conservatore, come del resto lo era stato anche Leo Frobenius. Ma anche tra questi autori engagés è forte il disincanto nei confronti della politica dell'epoca, caratterizzata da forti tensioni imperialiste guidate da interessi commerciali ed industriali.

«Tutto ciò che è spirituale deve avere un corpo sulla terra per essere attivo nella storia; questo corpo si costituisce da solo dove non si impedisce allo spirito di costruirlo. Quindi, per il momento, si tratta di eliminare gli ostacoli, di formare una comunità scelta e capace di mantenersi fedele. Se non si trovano le persone giuste in Germania per questo tentativo, e non si trovano presto, allora si può solo rinunciare al futuro della nostra patria: la Germania esisterà ancora per un po' di tempo forse, ma smetterà di vivere molto presto». ${ }^{32}$ 


\section{Il Tempo e la Storia}

30 La Germania, o per meglio dire le Germanie di de Lagarde, di Kantorowicz e di Frobenius, rinunciano alla loro attualità storica per pretenderne una ancora più decisiva: l'attualità di un progetto eterno, capace di plasmare il presente e determinare il futuro. Da una parte dunque le articolazioni temporali della Germania Segreta si estendono fino a comprendere tutti i tempi, mentre la sua stessa idea si situa fuori dal tempo. Questa dimensione ideale e sovratemporale della Germania Segreta è quella che ne determina il carattere sacrale.

"Che cosa è il sacro? È ciò che lega molte anime insieme. [...] Che cosa è la cosa più sacra? Ciò che oggi e per sempre fa sentire gli spiriti sempre più profondi, sempre e solo pochi. Si potrà ribaltare queste frasi [e con esse la domanda] e affermare che ciò che unisce le persone è il sacro. [.....] Se chiediamo della nazionalità tedesca dei nostri giorni, chiediamo degli ideali dei tedeschi dei nostri giorni. Non c'è risposta a questa domanda, perché tale ideale non esiste. Ed è per questo che non c'è la nazionalità tedesca'».33

31 Ciò che conferisce sacralità alla Germania Segreta è il suo essere invisibile, intangibile, ineffabile, irraggiungibile. Più che un ideale che si pone come meta di emulazione, essa è un Mistero, il cui potere è ancor più forte quando è circondato dal silenzio e dal pudore.

32 Già Friedrich Nietzsche, uno dei teorici di questa equazione concettuale tra il pudore (Scham), il Mistero e il Sacro, riconosceva questo meccanismo mitologico - per usare un'espressione di Jesi qui perfettamente calzante - ancora all'opera in alcuni dei più importanti ambiti della società moderna: nella gestione del potere, nell'ambito della religione, nel controllo dell'amore attraverso l'istituzione del matrimonio. ${ }^{34}$ In una parola, ciò di cui si ha pudore a parlare, ciò che si tiene nascosto e la cui esposizione alla luce del giorno procura disagio e vergogna oltre a determinarne una perdita di prestigio e valore: questo è per Nietzsche un Mistero. La Germania Segreta è per i suoi adepti un Mistero a tutti gli effetti. La retorica a contrario, gli argomenti e silentio adottati dai diversi teorici della teologia negativa della Germania segreta, richiamano quello che Furio Jesi definisce come discorso misterico.

33 Non è questa la sede per una discussione dei diversi aspetti della riflessione di Jesi sul mistero e i misteri antichi, tantomeno per rievocare tutti gli aspetti ancora irrisolti del dibattito sui Misteri storici nella storia delle religioni ed in filologia classica. Mi limito qui a richiamare solamente una delle chiavi di lettura proposte da Jesi, una delle meno note forse, e delle più raffinate a mio parere, come anche delle più provocatorie $\mathrm{e}$ stimolanti. Nel suo libro Il linguaggio delle Pietre, ${ }^{35}$ Jesi descrive la sapienza misterica, segreta e nascosta, che per definizione resta incomprensibile non solo ai profani, ma anche agli iniziati. Secondo questa interessante interpretazione, l'essenza stessa della condizione iniziatica consisterebbe proprio nel fatto che la sapienza misterica non richiede di essere veramente compresa. Questo avviene, come si pretende, perché questa sapienza è troppo profonda per essere compresa. Ma forse, e a questo arriva provocatoriamente la tesi di Jesi, il mistero è segreto ed incomprensibile poiché, semplicemente, non vi è nulla da comprendere. In linea con quella di Nietzsche, l'interpretazione di Jesi sembra suggerire che svelare un mistero sancisce già di per sé la fine del mistero, la fine del potere che il mistero detiene grazie alla sua aurea di pudore e silenzio; a maggior ragione se dietro il velo non c'è niente. 

però necessariamente interpretato in maniera negativa, sebbene sia un elemento da tenere ben presente quando si operi una critica di questo concetto e delle sue diverse articolazioni storiche e letterarie. L'assenza di un contenuto positivo è infatti un altro di quegli elementi che costituiscono quella che ho chiamato la "teologia negativa" dell'idea di Germania Segreta. Questa teologia negativa permette di declinare in molti altri modi il ricorso all'elemento misterico, non solo "esoterico", né semplicemente "misterioso", della Germania Segreta. Nel più mitologico dei libri scritti da Thomas Mann, Giuseppe e i suoi fratelli, nel Prologo - Discesa agli Inferi viene enunciata una definizione del Mistero: «il Mistero non conosce tempo, ma la forma di ciò che non ha tempo è il Qui e Ora». ${ }^{36}$ Concentriamoci ora in conclusione sulle articolazioni temporali del mitologema della Germania Segreta. Abbiamo parlato di inattualità e di rifiuto del presente come volgare, prosaico, inadeguato all'ideale. Al contempo però la Germania Segreta aspira ad una validità eterna, e, più concretamente, ad una incarnazione futura: «Tutto ciò che è spirituale deve avere un corpo sulla terra per essere attivo nella storia» diceva già de Lagarde. ${ }^{37}$ La Germania Segreta ha di fatto molteplici dimensioni temporali. La prima e più importante è quella del rapporto col passato. Come abbiamo visto, gli abitanti della Germania Segreta sono in primis i grandi uomini del passato. Quello della Germania Segreta potrebbe sembrare ad una prima valutazione un ideale nostalgico, rivolto ad un passato di grandezza, che non tornerà. Salvo che, da parte dei contemporanei, questo processo di legislazione della grandezza passata (Gesetzgebung der Grösse come la chiamerà Friedrich Nietzsche) ${ }^{38}$ costituisce un preciso atto fondativo. Si stabiliscono dei punti di partenza nel passato, delle figure di riferimento, dalle quali gettare delle prospettive verso il presente ed il futuro.

35 Letto in questa prospettiva storica si potrebbe spiegare ad esempio uno dei passaggi più controversi della Germania Segreta di Kantorowicz:

«La Germania Segreta è come un Giudizio Universale, e una risurrezione dei morti, sempre immediatamente vicina, presente. È realmente esistente e vera, come la morte. È la comunità segreta dei potenti e dei saggi, degli eroi e dei santi, dei carnefici e delle vittime che la Germania ha creato e che alla Germania si sono offerti».39

La nota forse troppo "orrorifica" di questo difficile passaggio potrebbe facilmente offrire il fianco alla celebre critica di Jesi verso la cosiddetta religio mortis della cultura tedesca. Con questo termine Jesi descriveva e condannava quello che a suo dire era il carattere dominante non solo delle élites militari dell'estrema destra (i teschi delle SS, il Viva la Muerte! dei falangisti spagnoli), ma anche delle élites culturali, degli amanti del mito e dell'antico, come lo stesso Kerényi, che indulgevano forse in buona fede, ma ancor più colpevolmente, in compiaciuti pensieri di morte. Un certo culto della morte avvicinava secondo Jesi diverse esperienze intellettuali, come quella di un Cesare Pavese, la "simpatia per la morte" di Thomas Mann, la pulsione di morte teorizzata da Sigmund Freud, fino ad arrivare all'essere per la morte di Martin Heidegger.

Ma se evitiamo una troppo scontata lettura "esoterico-orrorifica" di questo controverso passaggio di Kantorowicz e lo reinterpretiamo all'interno di una prospettiva storica, cosa che costituisce a mio parere la vera caratteristica dominante del pensiero di Kantorowicz, questo accenno alla resurrezione dei morti, al giorno in cui i morti cammineranno sulla terra, potrebbe apparire sotto una luce meno minacciosa. Per comprendere in che modo la Germania Segreta vedrà i 'suoi' morti camminare sulla terra, dobbiamo comprendere più a fondo le dimensioni temporali 
attraverso le quali si articola questo mitologema. All'incongruità di richiamarsi ad un passato che non c'è, per costruirlo in vista di un futuro ancora da produrre, si aggiunge il fatto che la Germania Segreta si ritiene al contempo antichissima (eterna e originaria) e giovanissima.

La giovinezza della Germania è quella della verspätete Nation di Helmut Plessner, quella nata per ultima nel grande processo di consolidamento nazionale degli stati europei, e che ha definito tardi i suoi lineamenti culturali (il suo Dante è nato a Francoforte nel 1749 e morto a Weimar nel 1832), spirituali (la Riforma luterana), linguistici (il dizionario dei Grimm). Questa giovane Germania era tanto nuova, che ancora doveva nascere al mondo, ancora doveva affacciarsi alla scena storica e politica, sulla quale da tempo le altre nazioni già agivano da protagoniste. A partire dal Kant del Saggio sull'lluminismo, ${ }^{40}$ fino ai teorici della dottrina letterario-politica della Rivoluzione Conservatrice di inizio secolo (tra i quali ritroviamo di nuovo George e Thomas Mann), che si rifacevano all'opera di storici come Kantorowicz, era opinione diffusa che la Germania sarebbe stata la prossima nazione a guidare una rivoluzione. Sulla natura di questa rivoluzione (una rivoluzione socialista? riformista? conservatrice? nazionalsocialista?) le ipotesi erano le più varie. L'elemento costante era però quello secondo il quale la Germania è un paese giovane, dalle forze spirituali ancora vergini e immature, che riceverà, una volta arrivato il suo momento, l'eredità di tutte le nazioni che l'hanno preceduta.

39 La "giovinezza" della Germania dello spirito, una Germania inattuale, fuori dal tempo, che eternamente si affaccia alla storia, permette di rinforzare quel parallelo, che già è stato un topos del classicismo tedesco e del primo romanticismo: l'equazione Greci/ Tedeschi. Anche il popolo greco era stato definito, dai sacerdoti di Sais, ${ }^{41}$ come anche da Winckelmann, ${ }^{42}$ un popolo giovane. Secondo Platone, i Greci non sono giovani solo perché si erano affacciati alla storia tardivamente, o almeno più tardi rispetto agli antichissimi Egizi. La loro giovinezza era garantita piuttosto dalla scarsa tendenza a conservare la memoria del passato, a vedere nella loro esistenza il prodotto del sapere accumulato dalle generazioni passate. Il popolo greco può essere antico quanto gli altri, ma non ha memoria di sé, non sente il peso del passato, non possiede storia e scienza, ma vive un eterno presente nel quale si affaccia ogni volta come se fosse appena nato.

40 Questa giovinezza però non ha nulla a che fare con la naïveté o l'originalità che Schiller ${ }^{43}$ pretendeva di attribuire ai Greci. La visione della Grecia dei cultori della Germania Segreta è molto cambiata rispetto all'epoca di Winckelmann e di Goethe. Non bisogna infatti dimenticare che nel frattempo c'è stato l'avvento di Nietzsche, che ha radicalmente cambiato la percezione del mondo antico. La Grecia di Nietzsche è sì un paese dalle energie ancora intatte, ma non certo un paese primitivo o semplice. I Greci "superficiali per profondità" 44 di Nietzsche sono circondati da grandi civiltà, da cui hanno saputo apprendere e di cui hanno assimilato caratteri e cultura, riuscendo a farli propri. Come i Greci ora i tedeschi, il popolo giovane, il popolo senza storia, è chiamato ad apprendere, andando alla scuola delle grandi civiltà europee, l'italiana, la francese, l'ebraica, per costruire una propria identità. Secondo de Lagarde, ${ }^{45}$ anche il carattere della Germania ideale è un carattere acquisito (Erworbenes) e non originale, che nega anzi questo concetto limite della creazione ex nihilo: le tappe spirituali della Germania Segreta sono frutto di conquiste spirituali.

41 Anche il rapporto con i cugini Greci obbedisce a questa legge di sviluppo. Come dice bene Kantorowicz, dando prova di un ammirevole virtuosismo nell'uso dell'ambiguità: 
«Certamente esiste una segreta parentela di sangue fra l'Ellade e la Germania, ma essa deve essere prima di tutto generata». ${ }^{46} \mathrm{Il}$ paradosso di un passato che non c'è ma che può e deve essere generato, ci porta a riflettere sui meccanismi della memoria e della produzione di modelli che hanno portato alla formulazione del mitologema della Germania Segreta. I meccanismi della memoria illustrati da Nietzsche nella II Abhandlung della Genealogia della Morale consentono all'uomo di costruirsi un passato dal quale derivare necessariamente, in modo da poter divenire un essere capace di promettere (versprechen), dunque capace di responsabilità (Verantwortlichkeit) rispetto al proprio futuro. ${ }^{47}$ Questa compresenza di determinismo ed anti-determinismo, di necessità e di arbitrio, ereditarietà e libertà, compaiono come componenti contraddittorie anche nel meccanismo della Germania Segreta.

42 Nel dispositivo della Germania Segreta si riconosce il funzionamento della macchina mitologica di Jesi, una struttura morfologica (un topos letterario in origine) capace di attrarre contenuti spirituali e di organizzarli all'interno di un'armatura di senso. In questo modo la Germania Segreta, situandosi in un luogo indeterminato tra il passato, il presente e il futuro, si costituisce come eterno punto di partenza per una nuova origine, per un'origine autentica. Il richiamo all'Ursprung è dunque del tutto fuorviante: non siamo qui di fronte ad una chimerica origine o ad un nostalgico compiacimento per un passato che non tornerà, ma abbiamo a che fare con un meccanismo fondativo $\mathrm{e}$ produttore di futuro. La Germania Segreta propone sì i modelli del passato, ma essi sono dei miti vivi ed attuali, capaci di indicare la via e fornire l'esempio. Si comprenderà ora forse più chiaramente il richiamo alla risurrezione dei morti della precedente citazione di Kantorowicz. Il giudizio universale di cui parla Kantorowicz è quello Jüngster Tag, l'ultimo giorno nel senso del giorno più giovane, che inaugura il Regno dei Cieli. Certo questa nuova origine, questa Ursprung ideale, è un'origine pretesa, finanche pretestuosa. Ma è proprio questo il punto interessante della produzione e rielaborazione di questo mitologema lungo quasi tutto un secolo, e certo non il secolo meno turbolento della storia della Germania: la convinzione che una cultura non possa nascere solamente secondo necessità, bensì secondo volontà.

\section{BIBLIOGRAFIA}

CHoIsY 1915: G. Choisy, L'Allemagne Secrète, Paris 1915.

CURTIUS 1948: E. R. Curtius, Europäische Literatur und lateinisches Mittelalter, Bern/München 1948.

FAVUZZI 2011: P. Favuzzi, «Simbolo, mito e verità storica. Albert Brackmann contro Ernst Kantorowicz», Archivio di Storia della Cultura, 24 (2011), 225-244.

FROBENIUS 1921-28: L. Frobenius, Atlantis: Volksmarchen und Volksdichtung Afrikas, Jena 1921-28, vol. 6 .

GERHARDT 2001: V. Gerhardt, «Übermensch», in Historisches Wörterbuch der Philosophie, Basel 2001, vol. $11,46$. 
GRÜNEWALD 2002: E. Grünewald, «La “Germania segreta” e il Terzo Reich» (ed. or. Wiesbaden 1982), trad. it., Filosofia dell'arte, 2 (2002), 159-173.

HEIDEGGER 2012: M. Heidegger, «Erläuterungen zu Hölderlins Dichtung (1936-1968)», in M. Heidegger, Gesamtausgabe, Frankfurt am Main 2012, vol. 4, 20.

VON HELLINGRATH (1982): N. von Hellingrath, La follia di Hölderlin (ed. or. München 1936), trad. it., Milano, 1982.

VON HELLINGRATH (1936): N. von Hellingrath, «Hölderlin und die Deutschen. Vortrag [1915]», in N. von Hellingrath, Hölderlin-Vermächtnis, München 1936.

JESI, KERÉNYI 1999: F. Jesi, K. Kerényi, Demone e mito. Carteggio 1964-1968, Macerata 1999.

JESI 1967: F. Jesi, Cultura di destra, Milano 1979.

JESI 1967: F. Jesi, Germania Segreta. Miti nella cultura tedesca del ‘900, Milano 1967.

JESI 2007: F. Jesi, L'accusa del sangue, Torino 2007.

JESI 1968: F. Jesi, Letteratura e mito, Torino 1968.

JESI 1978: F. Jesi, Il linguaggio delle pietre, Milano 1978.

KANT 1874: I. Kant, «Beantwortung der Frage: Was ist Aufklärung», Berlinische Monatsschrift 12 (1874), 481-494.

KANTOROWICZ 1997: E. Kantorowicz, « Das Geheime Deutschland [14. 11. 1933]», in R. L. Benson, J. Fried (eds.), Ernst Kantorowicz. Erträge einer Doppeltagung (Princeton/Frankfurt), Stuttgart 1997, 77-93.

KANTOROWICZ 1927: E. Kantorowicz, Kaiser Friedrich der Zweite, Berlin 1927.

KANTOROWICZ 1957: E. Kantorowicz, The King's Two Bodies. A Study in Mediaeval Political Theology, Princeton 1957.

KERÉNYI 1988: K. Kerényi, «Der Sprung: Nietzsche zwischen seinem Roman und seinem Evangelium», in K. Kerényi, Werke in Einzelausgaben, München 1988, V/2, 133-158.

VAN KOOTEN 2008: G. van Kooten, Paul's Anthropology in Context: The Image of God, Assimilation to God, and Tripartite Man in Ancient Judaism, Ancient Philosophy and Early Christianity, Tübingen 2008.

KossINNA 1902: G. Kossinna, «Die indogermanische Frage archäologisch beantwortet», Zeitschrift für Ethnologie» 34 (1902), 161-222.

DE LAGARDE 1878: P. de Lagarde, «Die Religion der Zukunft», in P. de Lagarde, Deutsche Schriften, Göttingen 1878, 217-255.

DE LAGARDE 1878: P. de Lagarde, «Über die gegenwärtige Lage des deutschen Reichs. Ein Bericht», in P. de Lagarde, Deutsche Schriften, Göttingen 1878, 67-154.

LANGBEHN 1890: J. Langbehn, Rembrandt als Erzieher, Leipzig 1890.

LASSON 1868: A. Lasson, Das Kulturideal und der Krieg, Berlin 1868.

MANN 1918: T. Mann, Betrachtungen eines Unpolitischen, Berlin 1918.

MANN 2006: T. Mann, Giuseppe e i suoi fratelli (ed. or. Berlin 1933-43), trad. it., Milano 2006.

MASSIMILLA 2005: E. Massimilla, «La “Germania Segreta” di Ernst Kantorowicz», in G. Furnari Luvarà, Filosofia e politica. Studi in onore di Girolamo Cotroneo, Soveria Mannelli 2005, 261-289. 
NIETZSCHE 1887: F. Nietzsche, Die Fröhliche Wissenschaft, Leipzig 1887.

NIETZSCHE 1889: F. Nietzsche, Götzen-Dämmerung, Leipzig 1889.

NIETZSCHE 1878: F. Nietzsche, Menschliches, Allzumenschliches, Chemnitz 1878.

NIETZSCHE 1969: F. Nietzsche, «Die Philosophie im tragischen Zeitalter der Griechen [1873]», in F. Nietzsche, Kritische Gesamtausgabe Werke, Berlin 1969, II/4, 218.

NIETZSCHE 1887: F. Nietzsche, Zur Genealogie der Moral, Leipzig 1887.

PLESSNER 1959: H. Plessner, Die verspätete Nation. Über die politische Verführbarkeit bürgerlichen Geistes [1935], Basel 1959.

POUND 1948: E. Pound, Pisan Cantos, New York 1948.

SANTINI 2011: C. Santini, «La reflexión sobre las religiones mistéricas en la filosofía de Nietzsche», Estudios Nietzsche (2011), 83-96.

SANTINI 2013: C. Santini, «La seduzione del mito. Furio Jesi, Thomas Mann e Karoly Kerényi», in S. Fornaro, D. Summa (a cura di), Eidolon. Saggi sulla tradizione classica, Bari 2013, 107-123.

SCHILLER 1795-96: F. Schiller, «Über naive und sentimentalische Dichtung», Die Horen (1795/96).

WINCKELMANN 1755: J. J. Winckelmann, Gedancken ilber die Nachahmung der Griechischen Wercke in der Mahlerey und Bildhauer-Kunst, Friedichstadt 1755.

WöLfFLIN 1905: H. Wölfflin, Die Kunst Albrecht Dürers, München 1905.

\section{NOTE}

1. Furio Jesi a Károly Kerényi, 2 Febbraio 1965 in JESI, KERÉNYI 1999.

2. Queste righe erano vergate in calce ad un articolo su Friedrich Nietzsche (KERÉNYI 1988, 133-158) che Kerényi inviò a Jesi qualche giorno dopo aver ricevuto la sua lettera del 2 Febbraio. Una risposta più circostanziata sarà invece inviata a Jesi in una lettera del 17 Maggio 1965.

3. A Norbert von Hellingrath si deve la riscoperta di Hölderlin, autore rimasto fino ad allora in secondo piano rispetto ai grandi classici di Weimar (se si eccettua la grande passione che ebbe per Hölderlin lo stesso Friedrich Nietzsche). L'impresa di Hellingrath si situa all'interno di una generale corrente di rivalutazione del patrimonio letterario ed artistico tedesco di fine Ottocento. Possono essere ricondotti a questo movimento di rinascita della cultura tedesca opere come il Rembrandt di Julius Langbehn (LANGBEHN 1890, il cui progetto, come è noto, mirava a presentare il celebre pittore fiammingo come campione dell'arte germanica), o il Dürer di Heinrich Wölfflin (wöLFFLIN 1905).

4. VON Hellingrath 1982, 24-25. «Wir nennen uns 'Volk Goethes', weil wir ihn als Höchsterreichbares unseres Stammes, als höchsten auf unseren Stamme Gewachsene, sehen in seiner reichen Munden Menschlichkeit. Ich nenne uns 'Volk Hölderlins', weil es zutiefst im deutschen Wesen liegt, dass sein innerster Glutkern unendlich weit unter des Schlacken-Kruste, die seine Oberfläche ist, nur in einem geheimen Deutschland zutage tritt; sich in Menschen äußert, die zum mindesten längst gestorben sein müssen, ehe sie gesehen werden, und Widerhall finden; in Werken, die immer nur ganz wenigen ihr Geheimnis anvertrauen, ja den meisten ganz schweigen, Nicht-Deutsche wohl nicht zugänglich sind; weil dieses geheime Deutschland so gewiss ist seines inneren Wertes oder so unschuldig unbekannt mit der eigenen Bedeutung, dass es gar keine Anstrengung macht, gehört, gesehen zu werden » (VON HELLINGRATH [1915] 1936, 124).

5. NIETZSCHE 1889, 49. 
6. CURTIUS 1948.

7. Lo Jahrbuch für die geistige Bewegung, giornale edito da Friedrich Wolters (1876-1930) e Friedrich Gundolf (1880-1931), può essere considerato l'organo di stampa e di diffusione delle idee esteticoletterarie degli intellettuali riuniti attorno a Stefan George. La rivista ha visto la pubblicazione di tre volumi tra il 1910 e il 1912 presso i tipi Otto von Olten di Berlino.

8. «Das alte Deutschland ist mit Nichten tot: aber es liegt viel tiefer und viel höher, als wo es der jetzige Reichskanzler und seine Freunde suchen. Was als klingendes Metall in den Glockenguss der Zukunft hineingeworfen werden wird, hat mit der Presse, und vollends mit einer Regierungspresse nichts zu tun. Hinter dem Pfluge und im Walde ist es zu finden» (DE LAGARDE 1878, 245).

9. Secondo David Bidussa nella sua introduzione all' Accusa del Sangue di Jesi, la macchina mitologica non è altro che una macchina desiderante, che si mette in moto laddove si esperisce un bisogno, una mancanza (JESI 2007, IX).

10. JESI 1979.

11. " Il y a l'Allemagne des politiciens et des militaires, il y a l'Allemagne des universités et des laboratoires, il y a celle des ingénieurs, celle de l'industrie, du commerce et de la finance, voire celle des écrivains et des artistes: elles s'apprennent peut être dans les livres. Il y a l'Allemagne des pensées et des gestes qui débordent tous les cadres et qu'aucune formule ne saurait enfermer, qui sont de toutes les catégories et de tous les métiers et professions et qui ne se voient ni ne s'expriment nulle part ou qui ne se voient que par accident et ne s'expriment que par oubli, qui enfin par leur nature même vont autrement loin dans le secret que les obscures manœuvres ou nos ennemis auront préparé et mené la Grande Guerre» (CHOISY 1915, 10-11, la traduzione è mia).

12. CHOISY 1915, 12.

13. CHOISY 1915, 12.

14. Solo per citare alcuni dei nomi più in vista di questa lista di signatari: lo storico dell'arte e curatore museale Wilhelm von Bode, lo scultore Adolf von Hildebrand, lo storico Karl Lamprecht, il pittore Max Liebermann, il filosofo Alois Riehl, il figlio di Richard Wagner il compositore Siegfried Wagner, il grande filologo Ulrich von Wilamowitz-Moellendorff e il filosofo e padre della psicologia Wilhelm Wundt.

15. «Car l'Allemagne essentielle, l'Allemagne qui demeure sous les formes qui passent et sous les hontes qui s'oublient, était déjà toute entière dans le chaos de l'époque ou la Germanie n'avait pas de nom, dans le mystère des forets que la main de l'homme fut si lente à éclaircir, et dans la désolation des marécages que le soleil n'a pas fini d'assécher, elle est née de la rencontre de ce mystère et de cette désolation et en elle les traits sont identifiables qui l'apparentent aux reptiles et aux grands fauves amis des bas-fonds et des épais fourrés» (CHoIsY 1915, 16, la traduzione è mia).

16. Il testo di questa conferenza esiste in due versioni con delle lievi differenze stilistiche: la prima conservata nel Nachlass di Kantorowicz presso il Leo Beck Institute di New York, l'altra nella biblioteca di Basel, nel fondo Edgar Salin. Poiché Kantorowicz aveva proibito la pubblicazione di quei testi che lui stesso non aveva rivisto per la stampa, questa conferenza venne pubblicata solo postuma [KANTOROWICZ 1997] e dunque Jesi non poté leggerla.

17. Come è noto Kantorowicz dovette infatti emigrare in America nel 1939, poco prima che le persecuzioni travolgessero la sua famiglia. Sua madre ed una cugina, Gertrud Kantorowicz, vennero infatti deportate a Theresienstadt nel 1942, dove morirono.

18. FROBENIUS 1921-28. Il racconto è citato in JESI 1968, 157-158.

19. POUND 1948, 427.

20. KANTOROWICZ 1997, 87.

21. KANTOROWICZ 1927. 
22. Questo approccio storiografico gli guadagnò, come è noto, l'aspra critica di Albert Brackmann, che accusò Kantorowicz di aver applicato l'immaginazione creatrice, la Einbildungskraft alla storiografia al solo scopo di creare un mito e non di fornire una trattazione storica affidabile nel senso della Darstellung rankiana. FAVuzzi 2011, 225-244.

23. Gustaf Kossinna, archeologo originario della Prussia Orientale (oggi Polonia), considerato uno dei padri degli studi sulla preistoria europea, fu uno dei propugnatori della tesi Indogermanica, secondo la quale i popoli germanici mitteleuropei costituirebbero il centro di una rete di diffusione culturale che si irradierebbe in tutta l'Europa e in gran parte del bacino del Mediterraneo. V. KOSSINNA 1902.

24. JESI, KERÉNYI 1999. Sulla questione della demonizzazione del mito nel carteggio tra Thomas Mann e Kerényi, e tra questi e Furio Jesi, vedi anche SANTINI 2013.

25. GERHARDT 2001. Su questo vedi anche VAN KOOTEN 2008.

26. «Deutschland ist kein geographischer aber auch kein in dem gewöhnlicher Sinne des Wortes Politisch politischer Begriff. Ein Vaterland gehört in die Zahl der ethischen Mächte und darum können seine Angelegenheiten nicht von Regierungsschichte sondern nur durch das ethische Pathos aller seiner Kinder besorgt werden» (DE LAGARDE 1878, 153, la traduzione è mia).

27. KANTOROWICZ 1957.

28. HEIDEGGER 2012, 20.

29. «Das Deutschland, welches wir lieben und zu sehen begehren, hat nie existiert, und wird vielleicht nie existieren. Das Ideal ist eben etwas, das zugleich ist und nicht ist. Es ist die im tiefsten Herzen der Menschen leuchtende Sonne, um welche unsere Gedanken und Kräfte, um Welche auch alle die Mittelpunkte schwingen, welche unser Leben umkreist, eine Sonne, deren Schein fahl und bleich wird, wann sie aus den Tiefen der Seelen an das Tageslicht emportaucht»» (DE LAGARDE 1878, 255, la traduzione è mia).

30. Plessner [1935] 1959.

31. MANN 1918.

32. «Alles Geistige muss auf der Erde einen Leib haben, um in der Geschichte tätig sein zu können; dieser Leib baut sich von selbst auf, wo man den Geist nicht hindert ihn zu bauen. Auf das Wegräumen der Hindernisse also kommt es vorläufig, auf die Bildung einen Zucht und Treue haltenden Gemeinde hauptsächlich an. Finden sich die Menschen für diesen Versuch in Deutschland nicht, und nicht bald, so können wir nur auf die Zukunft unseres Vaterlandes verzichten: Deutschland wird dann noch eine Weile existieren, zu leben wird es bald genug aufhören» (DE LAGARDE 1878, 242, la traduzione è mia).

33. «'Was ist Heilig? Das ist's, was viele Seelen zusammen bindet. [...] Was ist das Heiligste? Das, was heut und ewig die Geister, tiefer und tiefer gefühlt, immer nur einiger macht.' Man wird diese Sätze umdrehen um demzufolge behaupten dürfen, das Menschen Verbindende sei das Heilige. [...] Fragen mir nach der deutschen Nationalität unserer Tage, so fragen wir nach dem Ideale der Deutschen unserer Tage. Eine Antwort auf diese Frage gibt es nicht, denn ein solches Ideal ist nicht vorhanden. Und darum gibt es auch keine deutsche Nationalität» (DE LAGARDE 1878, 100. Il riferimento è alla lirica di Goethe, Vier Jahreszeiten - Erbst, v. 68-69).

34. NIETZSCHE 1878, I, 100. Sulla questione vedi SANTINI 2011.

35. JESI 1978.

36. MANN 2006.

37. DE LAGARDE $1878,242$.

38. NIETZSCHE 1969, II/4, 218.

39. KANTOROWICZ 1997, 80.

40. KANT 1874.

41. PLATON, Tim., 22b, 3-9.

42. WINCKELMANN 1755. 
43. SCHILLER 1795-96.

44. NIETZSCHE 1887, Vorrede 1886, XII.

45. DE LAGARDE $1878,138$.

46. KANTOROWICZ 1997, 88-89.

47. NIETZSCHE 1887, II, 41 sS..

\section{RIASSUNTI}

Questo articolo affronta il dibattito sulla "Germania Segreta" tra la fine dell'Ottocento e l'inizio del Novecento. Definisco la "Germania Segreta" innanzitutto come un topos letterario. Si tratta infatti di un fenomeno in origine eminentemente letterario, che si sviluppa all'interno di una cultura letteraria, quella tedesca e che all'interno di questa stessa cultura viene mobilitato come strumento critico per mutarsi poi un vero e proprio mitologema. Questa formula binaria si carica presto di significati che esulano dalla dimensione puramente finzionale e finanche estetica, e ambisce ad una validità più amplia, sia in campo storico, che sociale e politico, della cultura e della Weltanschauung tedesca. In questo articolo cercherò di definire i confini concettuali di quella che ho definito la "teologia negativa" della Germania Segreta, avvalendomi della guida metodologica di Jesi e ripercorrendo alcune delle tappe del suo sviluppo : da Paul de Lagarde, a Ernst Kantorowicz, a Gaston Choisy.

This article deals with the debate on "Secret Germany" between the end of the nineteenth and the beginning of the twentieth century. I define "Secret Germany" first of all as a literary topos. It is in fact at the origin an eminently literary phenomenon, that develops within a literary culture, the German one and that within this same culture is mobilized as a critical tool. This binary formula was soon however invested with meanings that went beyond the purely fictional and even aesthetic dimension, and aspired to a broader validity, both in the historical, social and political fields, as well as in the German culture and Weltanschauung. In this article I will try to define the conceptual boundaries of what I have called the "negative theology" of Secret Germany, using the methodological guide of Jesi and retracing some of the stages of its development: from Paul de Lagarde, to Ernst Kantorowicz, to Gaston Choisy.

\section{INDICE}

Parole chiave : mito, Jesi Furio, Germania Segreta, Kantorowicz Ernst, topos letterario

Keywords : myth, Jesi Furio, Secret Germany, Kantorowicz Ernst, literary topos

\section{AUTORE}

\section{CARLOTTA SANTINI}

CNRS / ENS - UMR 8547 Pays Germaniques / Transferts culturels 
Ecole Normale Superieure,

45, Rue d'Ulm,

75005 Paris - France

carlottasantini(at)hotmail.it 